\title{
Translational medicine in dysphagia treatment
}

\author{
Printza $A^{*}$, Constantinidis J and Triaridis $S$ \\ $1^{\text {st }}$ Otolaryngology Department, Medical Department, School of Health Sciences, Aristotle University of Thessaloniki, 54124, Thessaloniki, Greece
}

\begin{abstract}
Dysphagia is prevalent in the general population, associated with significant morbidity and mortality and serious socioeconomic implications. There is extensive recent research in all aspects of dysphagia management. The aim of this review is to highlight top issues related to translational science in the field of dysphagia. Recent literature on dysphagia screening, diagnosis and treatment was reviewed to critically appraise areas of potential research translation to clinical practice and identify issues in need for further investigation before translation into practice or precision medicine is possible.
\end{abstract}

A key finding of our review is that an obstacle to research translation is the variability in the definition of dysphagia in research (swallowing safety, effectiveness, or both) and the diversity of phenotypes of most diseases related to dysphagia.

Review of the literature on screening, clinical evaluation, and instrumental tests documented the need for further improvement of patient-reported outcome measures and screening tests to meet adequate methodological criteria. Another finding is the ongoing research for further standardization, evaluation of the reliability of ratings and comparison of the diagnostic accuracy of Videofluoroscopy Swallowing Study and Fiberoptic Endoscopic Evaluation of Swallowing.

Translational research has shown potential for a future change in managment strategies for head and neck cancer, to the direction of precision medicine and there is emerging evidence regarding the quantitative evaluation of head and neck cancer treatment-related dysphagia. Evidence is accumulating to support the effectiveness of strength training treatment protocols. Critical appraisal of the existing literature highlights methodological issues in need for further investigation regarding the application of non-invasive brain stimulation on dysphagic patients.

Diversity makes translational medicine in dysphagia difficult. Translation of the existing findings to clinical practice is accentuated by the extensive recent research in the field of dysphagia, pointing to an emerging potential to support precision medicine in the near future.

Dysphagia is a common symptom, associated with significant morbidity and mortality. Its prevalence in the general population has been reported to range from $13.5 \%$ to $22.6 \%$ [1-3]. It may result in dehydration, malnutrition, aspiration pneumonia and even death [4]. Dysphagia can have a profound impact on a patient's life and also has severe socioeconomic implications [5]. Dysphagia is highly prevalent in the older population [6,7]. It is also associated with stroke [4,8,9], Parkinson's disease [9-10], multiple sclerosis [11-13], head and neck cancer [14], neuromuscular diseases, trauma, dementia, gastroesophageal reflux and oesophageal diseases [5,7]. The exact epidemiological numbers remain poorly defined. This is partly due to the fact that dysphagia is a symptom and diversity of phenotypes is a typical feature of most diseases related to dysphagia. Diversity makes translational medicine and precision medicine in dysphagia difficult. There is extensive research over the past decade in all aspects of dysphagia prevention, diagnosis, and therapy. The aim of this review is to highlight top research issues related to translational science in the field of oropharyngeal dysphagia. Recent literature reporting on dysphagia screening, diagnosis and treatment was reviewed to critically appraise areas of potential translation of the existing research into clinical practice and identify issues in need for further investigation before translation into practice or precision medicine is possible.

Dysphagia is assessed by screening procedures, clinical evaluation /examination, and instrumental tests. Screening tests aim to identify the likelihood of a swallowing impairment in persons not previously identified as dysphagic. Clinical diagnostic tests serve to confirm the presence, location, and severity of swallowing impairment. Instrumental examinations utilize technology to observe swallowing via endoscopy, or dynamic imaging or measure dysphagia physiology and offer a detailed assessment of swallowing dysfunction and of the effectiveness of various eating strategies.

The combination of the three approaches is an established practice in the clinical setting. An obstacle to the process of translating research findings to clinical practices is the variability in the definition of dysphagia in research. Some articles define dysphagia on the basis of airway safety alone. A diagnostic test can have higher sensitivity for the diagnosis of aspiration than for the diagnosis of milder dysphagia events that compromise swallowing efficiency like pharyngeal food pooling.

All assessment tests need to be reliable, valid, and feasible, of high sensitivity and specificity. A paradigm of integration of research findings to clinical screening practice is stroke-related dysphagia. Evidence that early detection of dysphagia reduces pulmonary complications, length of hospital stay, and overall health care costs [15] led to the development of specific guidelines for screening stroke patients for dysphagia with validated tools [16]. Although stroke dysphagia

*Correspondence to: Athanasia Printza, MD, MSc, PhD, Assistant Professor of Otolaryngology, $1^{\text {st }}$ Otolaryngology Department, School of Health Sciences, Aristotle University of Thessaloniki, 54124, Thessaloniki, Greece, E-mail: nan@med.auth.gr

Key words: dysphagia, translational research, dyshagia screening, Fiberoptic Endoscopic Evaluation of Swallowing, strength training, brain stimulation

Received: May 21, 2018; Accepted: May 28, 2018; Published: May 29, 2018 
screening guidelines are in effect for many years a recent systematic review critically appraised 35 published screening protocols, of which only two met adequate methodological criteria [17]. The difficulty in developing tests of high-quality overall is depicted to the fact that 34 dysphagia related Patient-reported outcome measures were published from 1987 until $2014[18,19]$.

The Videofluoroscopy Swallowing Study (VFSS) and Fiberoptic Endoscopic Evaluation of Swallowing (FEES) are well established instrumental examinations of swallowing. Although a reporting scale for the main signs of dysphagia (bolus penetration and aspiration) has been published 20 years ago [20] and is systematically used in clinical practice and research, there is ongoing research for further standardization [14,21], evaluation of the reliability of ratings [22] and comparison of the diagnostic accuracy of VFSS and FEES $[23,24]$.

Regarding genetic conditions related dysphagia, there is limited research relating endotypes and dysphagia phenotypes to guide evidence-based decisions for screening, diagnostic evaluations and treatment [25].

A common cause of dysphagia is head and neck cancer. Squamous cell carcinoma of the head and neck (SCCHN) is heterogeneous both clinically and biologically. Translational research has shown potential for a future change in management strategies for SCCHN to the direction of precision medicine [26]. There is emerging evidence regarding the quantitative evaluation of head and neck cancer treatment-related dysphagia $[14,26]$. These findings are valuable for the development of a personalized treatment deintensification paradigm.

Currently the management of dysphagia focuses on the prevention of complications while any natural recovery takes place and the implementation of exercise programs. Studies in healthy individuals and dysphagic populations inform evidence-based decisions about treatment protocols. Most therapeutic exercise programs used to treat dysphagia include isometric lingual strength training. Only recently evidence is accumulating to support the effectiveness of such treatment protocols and guide patient selection, type and length of training regimes [27].

Recently there has been interest in the role of neuromodulation to treat dysphagia. The central neural processing of swallowing has been extensively investigated over the last two decades. Non-invasive brain stimulation techniques were used initially to explore the physiological swallowing mechanisms in healthy subjects $[28,29]$. Later on a number of studies investigated the efficacy of transcranial magnetic stimulation (TMS) and transcranial direct current stimulation (tDCS) in the management of neurogenic dysphagia [30-32]. To date it is difficult to draw conclusions for the efficacy of these techniques. Reviews of the few relevant randomised controlled trials in the literature showed a moderate significant positive overall effect. There were many methodological differences across research studies, including patient characteristics, accounting for the still existing controversy as to which site to stimulate, strength and duration of stimulation [31,32]. Further research is needed to investigate the application of non-invasive brain stimulation on dysphagic patients prior to the translation into clinical practice for dysphagia rehabilitation.

Diversity makes translational medicine in dysphagia difficult. Translation of the existing findings to clinical practice is accentuated by the extensive recent research in the field of dysphagia, pointing to an emerging potential to support precision medicine in the near future.

\section{Authorship}

All the authors made significant contribution to the study. All the authors have read and approved the final version of the manuscript.

\section{Funding information}

The authors confirm that they have no financial interests in relation to the work. This research received no specific grant from any funding agency in the public, commercial, or not-for-profit sectors.

\section{Conflicts of interest}

The authors Athanasia Printza, MD, MSc, PhD, Jannis Constantinidis, $\mathrm{MD}, \mathrm{PhD}$, Stefanos Triaridis, $\mathrm{MD}, \mathrm{MSc}, \mathrm{PhD}$, declare that there is no conflict of interest related to this paper.

\section{Ethical approval}

This study was performed in accordance with the ethical standards of the institutional and/or national research committee and with the 1964 Helsinki declaration and its later amendments.

\section{References}

1. Wilkins T, Gillies R, Thomas A, Wagner P (2007) The prevalence of dysphagia in primary care patients: a Hames Net Research Network study. J Am Board Fam Med 20: $144-150$

2. Locke GR,Talley NJ, Fett SL, Zinsmeister AR, Melton LJ, et al. (1997) Prevalence and clinical spectrum of gastroesophageal reflux: a population-based study in Olmsted County, Minnesota. Gastroenterology. 112: 1448-1456.

3. Eslick G, Talley N (2008) Dysphagia: epidemiology, risk factors and impact on quality of life - a population-based study. Aliment Pharmacol Ther 27: 971-979.

4. Martino R, Foley N, Bhogal S, Diamant N, Speechley M, et al. (2005) Dysphagia after stroke: incidence, diagnosis, and pulmonary complications. Stroke 36: 2756-2763.

5. Ekberg O, Hamdy S, Woisard V, Wuttge-Hannig A, Ortega P, et al. (2002) Social and psychological burden of dysphagia: its impact on diagnosis and treatment. Dysphagia 17: 139-146.

6. Lindgren S, Janzon L (1991) Prevalence of swallowing complaints and clinical findings among 50-79-year-old men and women in an urban population. Dysphagia 6: 187-192.

7. Wirth R, Dziewas R, Beck A, et al. (2016) Oropharyngeal dysphagia in older persons - from pathophysiology to adequate intervention: a review and summary of an international expert meeting. Clin Interv Aging 23: 189-208.

8. Mann G, Hankey G, Cameron D (2000) Swallowing disorders following acute stroke: prevalence and diagnostic accuracy. Cerebrovasc Dis 10: 380-386.

9. Takizawa C, Gemmell E, Kenworthy J, Speyer R (2016) A Systematic Review of the Prevalence of Oropharyngeal Dysphagia in Stroke, Parkinson's Disease, Alzheimer's Disease, Head Injury, and Pneumonia. Dysphagia 31: 434-441.

10. Kalf J, de Swart B, Bloem B, Munneke M (2012) Prevalence of oropharyngeal dysphagia in Parkinson's disease: a meta-analysis. Parkinsonism Relat Disord 18: 311 315 .

11. Printza A, Kalaitzi M, Bakirtzis C, Nikolaidis I, Proios H, et al. (2018) Reliability and Validity of the DYMUS Questionnaire for the assessment of dysphagia in Multiple Sclerosis (Greek version) and proposed modification. Mult Scler Relat Disord

12. Guan X, Wang H, Huang H, Meng L (2015) Prevalence of dysphagia in multiple sclerosis: a systematic review and meta-analysis. Neurol Sci 36: 671-681.

13. Calcagno P, Ruoppolo G, Grasso MG, De Vincentiis M, Paolucci S, et al. (2002) Dysphagia in multiple sclerosis - prevalence and prognostic factors. Acta Neurol Scand 105: 40-43.

14. Xinou E, Chryssogonidis I, Kalogera-Fountzila A, Panagiotopoulou-Mpoukla D, Printza A, et al. (2018) Longitudinal Evaluation of Swallowing with Videofluoroscopy in Patients with Locally Advanced Head and Neck Cancer After Chemoradiation. Dysphagia.

15. Hinchey J, Shephard T, Furie K, et al. (2005) Formal dysphagia screening protocols prevent pneumonia. Stroke 36: 1972-1976. 
16. National Guideline Clearinghouse USA «NGC:009281 2001 OCT (REV 2012 JUL) Diagnosis and initial treatment of ischemic stroke. Institute for Clinical Systems Improvement».

17. Schepp S, Tirschwell D, Miller R, Longstreth WJ (2012) Swallowing screens after acute stroke: a systematic review. Stroke 43: 869-871.

18. Patel DA, Sharda R, Hovis KL, et al. (2017) Patient-reported outcome measures in dysphagia: a systematic review of instrument development and validation. Dis Esophagus 1: 1-23.

19. Printza A KA, Pavlidou E, Triaridis S, Constantinidis J (2018) Reliability and Validity of the Eating Assessment Tool-10 (Greek adaptation) in neurogenic and head and neck cancer related oropharyngeal dysphagia. Eur Arch Otorhinolaryngol.

20. Rosenbek JC, Robbins JA, Roecker EB, Coyle JL, Wood JL (1996) A penetrationaspiration scale Dysphagia 11: 93-98.

21. Martin-Harris B, Brodsky M, Michel Y (2008) MBS measurement tool for swallow impairment-MBSimp: establishing a standard. Dysphagia 23: 392-405.

22. Pisegna J, Borders JC, Kaneoka A, Coster W, Leonard R, et al. (2018) Reliability of Untrained and Experienced Raters on FEES: Rating Overall Residue is a Simple Task. Dysphagia.

23. Giraldo-Cadavid L, Leal-Leaño L, Leon-Basantes G, et al. (2017) Accuracy of endoscopic and videofluoroscopic evaluations of swallowing for oropharyngeal dysphagia. Laryngoscope 1-127

24. Coffey M, Tolley N, Howard D, Drinnan M (2018) An Investigation of the Postlaryngectomy Swallow Using Videofluoroscopy and Fiberoptic Endoscopic Evaluation of Swallowing (FEES). Dysphagia.
25. Raginis-Zborowska A, Pendleton N, Hamdy S (2016) Genetic determinants of swallowing impairment, recovery and responsiveness to treatment. Curr Phys Med Rehabil Rep 4: 249-256.

26. Quon H, Hui X, Cheng Z, et al. (2017) Quantitative Evaluation of Head and Neck Cancer Treatment-Related Dysphagia in the Development of a Personalized Treatment Deintensification Paradigm. Int J Radiat Oncol Biol Phys 99: 1271-1278.

27. McKenna V, Zhang B, Haines M, Kelchner L (2017) A Systematic Review of Isometric Lingual Strength-Training Programs in Adults With and Without Dysphagia. Am J Speech Lang Pathol 26: 524-539.

28. Hamdy S (2003) The organisation and re-organisation of human swallowing motor cortex. Clin Neurophysiol 31: 204-210.

29. Michou E, Raginis-Zborowska A, Watanabe M, Lodhi T, Hamdy S, et al. (2016) Repetitive Transcranial Magnetic Stimulation: a Novel Approach for Treating Oropharyngeal Dysphagia. Curr Gastroenterol Rep 8:10.

30. Simons A, Hamdy S (2017) The Use of Brain Stimulation in Dysphagia Management Dysphagia 32: 209-215.

31. Yang S, Pyun S, Kim HJ (2015) Effectiveness of Non-invasive Brain Stimulation in Dysphagia Subsequent to Stroke: A Systemic Review and Meta-analysis. Dysphagia 30: 383-391.

32. Pisegna J, Kaneoka A, Pearson WJ, Kumar S, Langmore S (2016) Effects of noninvasive brain stimulation on post-stroke dysphagia: A systematic review and metaanalysis of randomized controlled trials. Clin Neurophysiol 127: 956-968.

Copyright: ( 2018 Printza A. This is an open-access article distributed under the terms of the Creative Commons Attribution License, which permits unrestricted use, distribution, and reproduction in any medium, provided the original author and source are credited. 